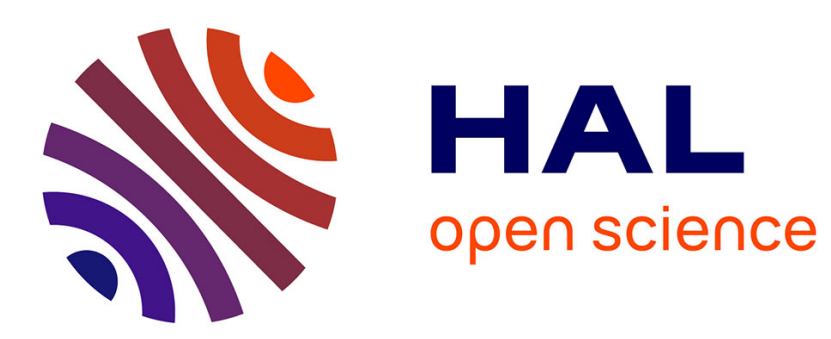

\title{
Effets et limites du commerce équitable Fairtrade sur les producteurs de café arabica d'une région de piémont andin, au Pérou
}

Elise Bouedron, Hubert Cochet, Paul Belchi

\section{- To cite this version:}

Elise Bouedron, Hubert Cochet, Paul Belchi. Effets et limites du commerce équitable Fairtrade sur les producteurs de café arabica d'une région de piémont andin, au Pérou. Revue internationale des études du développement, 2019. hal-02533289

HAL Id: hal-02533289

https://hal.science/hal-02533289

Submitted on 6 Apr 2020

HAL is a multi-disciplinary open access archive for the deposit and dissemination of scientific research documents, whether they are published or not. The documents may come from teaching and research institutions in France or abroad, or from public or private research centers.
L'archive ouverte pluridisciplinaire HAL, est destinée au dépôt et à la diffusion de documents scientifiques de niveau recherche, publiés ou non, émanant des établissements d'enseignement et de recherche français ou étrangers, des laboratoires publics ou privés. 


\title{
Effets et limites du commerce équitable Fairtrade sur les producteurs de café arabica d'une région de piémont andin au Pérou
}

\author{
Elise Bouëdron, Hubert Cochet et Paul Belchi
}

\section{Résumé}

À travers l'analyse diagnostic du système agraire, cette étude a permis d'identifier à quelles conditions certains systèmes de production du piémont andin de San Ignacio au Pérou ont pu s'insérer dans le commerce équitable Fairtrade. L'analyse technico-économique des systèmes de production fournit des données de performance agronomique et de revenus agricoles des ménages et permet une comparaison chiffrée entre producteurs intégrés au commerce équitable Fairtrade et les autres. Les membres des coopératives certifiées ont vu augmenter significativement leur revenu, aujourd'hui entre 15 à $50 \%$ supérieur à des producteurs non certifiés, sans pour autant gommer les différences de revenus agricoles qui restent dépendants de l'accès à la terre, ni la spécialisation caféière. Des éléments clés ont été identifiés pour assurer la performance et la durabilité de la production de café certifiée Fairtrade, tel que l'appui à la diversification culturale, un encadrement et un ciblage recentré sur les plus petits producteurs.

Mots clés : impact, Fairtrade, Pérou, café, agriculture comparée 


\section{Introduction}

Depuis ses débuts dans les années 1960, le commerce équitable cherche à rééquilibrer les rapports commerciaux entre pays producteurs et consommateurs. Produit emblématique du commerce équitable, le café représente plus de $50 \%$ du chiffre d'affaire des produits labellisés Fairtrade en France. Parmi les labels du commerce équitable, Fairtrade est le plus développé dans la filière café avec 537 coopératives certifiées en 2016, face environ 121 pour les certifications SPP, Fair For Life et BioPartenaire, dont certaines sont également certifiées Fairtrade (BASIC, 2019). C'est à partir des années 2000, alors que les volumes commercialisés en Fairtrade augmentent considérablement, que la demande de justification de l'impact du commerce équitable de la part des consommateurs, décideurs et acteurs de la filière s'accroît. Le dispositif de mesure d'impact du commerce équitable, développé par Eberhart en 2007, propose un cadre d'évaluation des effets du commerce équitable sur les ménages, les organisations de producteurs et le développement local. Dans cette étude, nous nous concentrerons sur les effets sur les ménages (type de famille, influence sur les revenus et les conditions de vie, l'investissement et la diversification agricole).

Le Pérou est le plus important exportateur de café Fairtrade en volume (Fairtrade International, 2017). Les coopératives de la région étudiée ${ }^{1}$, le piémont andin de San Ignacio au Nord Pérou, accèdent à la certification à partir de 2005 et regroupent aujourd'hui environ $40 \%$ des producteurs de café de cette région qui comporte environ 8500 producteurs. Des exportateurs privés ont, eux aussi, créé des groupements de producteurs certifiés bio et «durables » (RainForest Alliance, Café Practice, etc.), auxquels ils fournissent des services semblables à ceux des coopératives. Près de quinze années après, cet article s'intéresse à l'impact de la certification sur les producteurs de cette région. ${ }^{2}$

Notre étude est guidée par l'hypothèse que Fairtrade offre des revenus plus élevés aux producteurs membres, mais que l'intégration aux filières certifiées dépend des ressources préalables des familles. L'étude s'intéresse aussi au rôle du prix minimum sur les revenus des producteurs.

\section{Une analyse du système agraire pertinente pour apprécier la contribution globale du label Fairtrade}

Des ouvrages de référence comme celui Virginie Diaz Pedregal (2006) ou celui de Valérie Nelson (2017) permettent d'appréhender, à travers une riche revue des études existantes, les effets économiques et sociaux du commerce équitable, mais ne proposent pas de données

\footnotetext{
${ }^{1}$ Les coopératives les plus importantes de la région d'étude : Cenfrocafé (3000 membres, 12500 tonnes de café vert exporté), Casil (200 membres, 650 tonnes de café vert exporté), COOPAFSI (350 membres, 1100 tonnes de café vert exporté), Aprocassi (500 membres).

${ }^{2}$ Cette recherche a été commanditée par Max Havelaar France et le Réseau Latino-américain et caribéens de commerce équitable des petits producteurs et travailleurs. Elle repose sur un travail de terrain approfondi réalisé de mars à août 2018 par le premier auteur de cet article (Bouëdron, 2018) dans la région de San Ignacio.
} 
chiffrées sur les retombées économiques sur les revenus des ménages. La synthèse d'Oya et al. (2018) analyse les résultats économiques de plusieurs études et trouve un effet statistiquement nul des productions certifiées sur les revenus des ménages. Notre étude montre un revenu agricole 15 à $50 \%$ plus élevé pour les producteurs membres d'une coopérative certifiée. La littérature scientifique montre que l'impact du Fairtrade dépend, d'une part, de la valeur du cours mondial par rapport au prix minimum, et d'autre part des conditions d'intégration au marché (Darko, 2017). En effet, les études menées, entre 2000 et 2004, alors que le cours mondial était très en-deçà du prix minimum Fairtrade, ont montré des résultats positifs du commerce équitable sur les prix et sur les revenus des producteurs (Valkila, 2009 ; Mendez, 2010). En revanche, sur la période 2007-2008, alors que les cours étaient supérieurs au prix minimum, les résultats n'étaient pas significatifs (Ruben, 2011). Notre étude se base sur les prix pratiqués entre 2012 et 2017, période où les cours ont été parfois supérieurs, parfois inférieurs au prix minimum Fairtrade, ce qui permet de diminuer le biais précédemment cité. De plus, les coopératives interrogées sont intégrées au système Fairtrade depuis 15 ans, leur part de contrats aux conditions Fairtrade s'est stabilisée entre 30 et $70 \%$ selon la taille de la coopérative, ces dernières sont donc relativement bien intégrées au marché.

Les études précédemment menées comparent les revenus de producteurs certifiés Fairtrade à ceux des membres de coopératives non Fairtrade - en système conventionnel, en agriculture biologique ou avec un label privé «durable» (Ruben, 2010). Cependant, les coopératives du piémont andin de San Ignacio cumulent les trois types de certifications, ce qui leur permet de vendre l'ensemble de leur production au-dessus du prix du marché mondial. La comparaison est donc réalisée avec des producteurs non-membres des coopératives, certains certifiés « agriculture biologique » ou « durable » par l'intermédiaire d'un exportateur privé, et d'autres « indépendants » en système conventionnel. On mesure à la fois l'effet des filières certifiées sur le revenu des producteurs, ainsi que l'effet de l'appartenance à une coopérative certifiée Fairtrade, comparé à un groupe formé par un exportateur privé certifié biologique et durable.

Pour appréhender l'impact de la certification sur les exploitations agricoles qui en ont bénéficié, à l'échelle de cette petite région agricole, nous avons développé une approche d'agriculture comparée (Cochet et al., 2007, Cochet 2011). Un grand soin a été apporté à l'étude diachronique du système agraire pour identifier, à la fois, les transformations contemporaines des unités de production qui ont bénéficié de cette certification et les trajectoires de celles, tout aussi nombreuses, qui n'ont pas été intégrées dans les circuits de certification. Pour ce faire, une trentaine d'entretiens historiques approfondis ont été conduits et ont permis de mettre en lumière les dynamiques d'évolution et les processus de différenciation de la situation agraire (annexe 2). Cette approche historique couplée à une analyse détaillée du paysage et des conditions écosystémiques dans lesquelles opèrent les agriculteurs nous a permis d'identifier les principaux types d'unités de production opérant aujourd'hui dans la région (Cochet et Devienne, 2006).

Pour appréhender cette diversité de situations, un échantillon raisonné d'exploitations a ensuite été constitué de façon à embrasser les principaux types d'exploitations. Une typologie de systèmes de production a ainsi été élaborée, chaque système de production représentant un ensemble d'exploitations qui ont le même accès aux ressources que sont la terre, la maind'œuvre et l'équipement et qui mettent en œuvre les mêmes combinaisons de systèmes de cultures et/ou d'élevage (Cochet et Devienne, op. cit.). Trois à quatre enquêtes technicoéconomiques ont été menées pour chacun des seize systèmes de production préalablement identifiés (annexe 1). C'est ainsi qu'une cinquantaine de visites d'exploitation et 
d'entretiens technico-économiques approfondis ont été réalisés auprès des producteurs de la région (démarche complétée par des enquêtes auprès d'acteurs de la filière).

Les entretiens approfondis réalisés auprès des producteurs avaient à la fois pour objectif de comprendre le processus de production lui-même dans tous ces aspects techniques (ressources utilisées, itinéraires techniques et conduite, performances agronomiques) et de rassembler les données nécessaires à la mesure des résultats économiques, notamment du revenu agricole. Le revenu agricole est défini comme suit : il s'agit de la valeur monétaire du produit brut (autoconsommation comprise), diminué des consommations intermédiaires, de la dépréciation du capital fixe, du loyer versé pour les terres prise en location, des salaires versés à des tiers (avantages en nature compris) et d'éventuelles taxes et intérêts versés sur le capital emprunté. Il prend en compte tout ce qui est produit sur l'exploitation : les cultures alimentaires, le café et l'élevage. Le revenu agricole ne prend pas en compte le travail salarié dans les autres exploitations ou le travail dans un autre secteur économique. L'étude montre que la plupart des producteurs moyens (2-3.5 ha) n'ont pas d'autre activité économique à part la récolte du café dans les autres exploitations, ce qui représente un revenu 10 fois moins important que leur revenu agricole. Les petits producteurs $(0.5-2 \mathrm{ha})$ travaillent aussi comme ouvrier agricole et dans d'autres secteurs économiques, ce qui peut représenter plus de $50 \%$ du revenu total des ménages. L'étude propose de comparer le revenu agricole au seuil de survie calculé avec les données spécifiques au terrain, pour mieux identifier l'effet sur les conditions de vie des ménages.

\section{Une histoire locale du café : ressources,} politiques et marchés en perspective

\section{Le café arabica : moteur du développement économique de la région}

Le piémont andin de San Ignacio au nord du Pérou (figures 1 et 2) correspond à une zone de forêt tropicale située entre 700 et $2300 \mathrm{~m}$ d'altitude. Le café y est cultivé manuellement; il est la principale culture de rente et occupe la quasi-totalité de la surface agricole utile. Il est cultivé en association avec des plantes vivrières les premières années de son développement (manioc, bananier plantain, maïs, etc.), puis en association avec des arbres d'ombrage (agroforesterie à ombrage faible à moyen selon l'altitude et le degré d'ensoleillement : 50 à $100 \mathrm{arbres} / \mathrm{ha}$, fruitiers et pour le bois d'œuvre). L'essentiel de la production de café de la région est aujourd'hui transformée par voir humide ${ }^{3}$, et chaque producteur possède son matériel de transformation des cerises en café parche (non dépulpé). Dans les fonds de vallées, l'humidité excessive empêche le bon développement du café mais est propice à l'installation de prairies pour l'élevage bovin mixte dont les produits sont à destination du marché local. Les familles élèvent aussi pour leur consommation personnelle des volailles, des cochons d'inde et/ou des cochons, nourris généralement au grain de maïs, à la banane plantain et au manioc produits sur l'exploitation.

Pendant la deuxième moitié du $\mathrm{XX}^{\mathrm{e}}$ siècle, la région de San Ignacio, jusqu'alors très faiblement peuplée, connait un boom démographique ${ }^{4}$ avec l'augmentation du prix relatif du café (figure 3), liée au développement de la consommation en Europe. Des migrants affluent à partir des

\footnotetext{
${ }^{3}$ Les cerises de café sont dépulpées, les grains sont fermentés, séchés pour obtenir un café en parche. Le café en parche sera déparché en usine pour obtenir le café vert exportable.

4 La population est multipliée par 15 entre 1940 et 2008 (Girot, 1984 et statistiques de la municipalité de San Ignacio).
} 
années 1950 pour venir installer, par abattis-brûlis, une petite surface en café et en cultures vivrières. Les témoignages - recueillis auprès des personnes âgées qui ont vécu cette période permettent de reconstituer à grands traits ce développement de l'économie caféière. Les familles ne cultivaient qu'une seule variété de café, l'Arabica typica, et sa gestion demandait relativement peu de travail, hormis la récolte. Les cultures vivrières occupaient encore une part importante de l'assolement et du calendrier cultural. Les migrations continuent au cours du $\mathrm{XX}^{\mathrm{e}}$ siècle et l'agriculture gagne peu à peu sur la forêt primaire. Les systèmes de production se différencient alors selon la surface que les familles peuvent cultiver (main-d'œuvre familiale disponible) et le type de terrain auxquels elles ont accès (compatible ou non avec la culture du café ou des cultures vivrières). Les coopératives, formées grâce à un cadre législatif favorable (Dans les années 1960, Belaunde édicte la Loi générale des coopératives), et les petits commerçants privés achètent le café en coque aux producteurs (voie sèche ${ }^{5}$ ), le pilent et vendent le café vert aux grossistes de la côte. La production et la vente du café stimulent la croissance de la ville de San Ignacio et le développement des autres activités économiques.

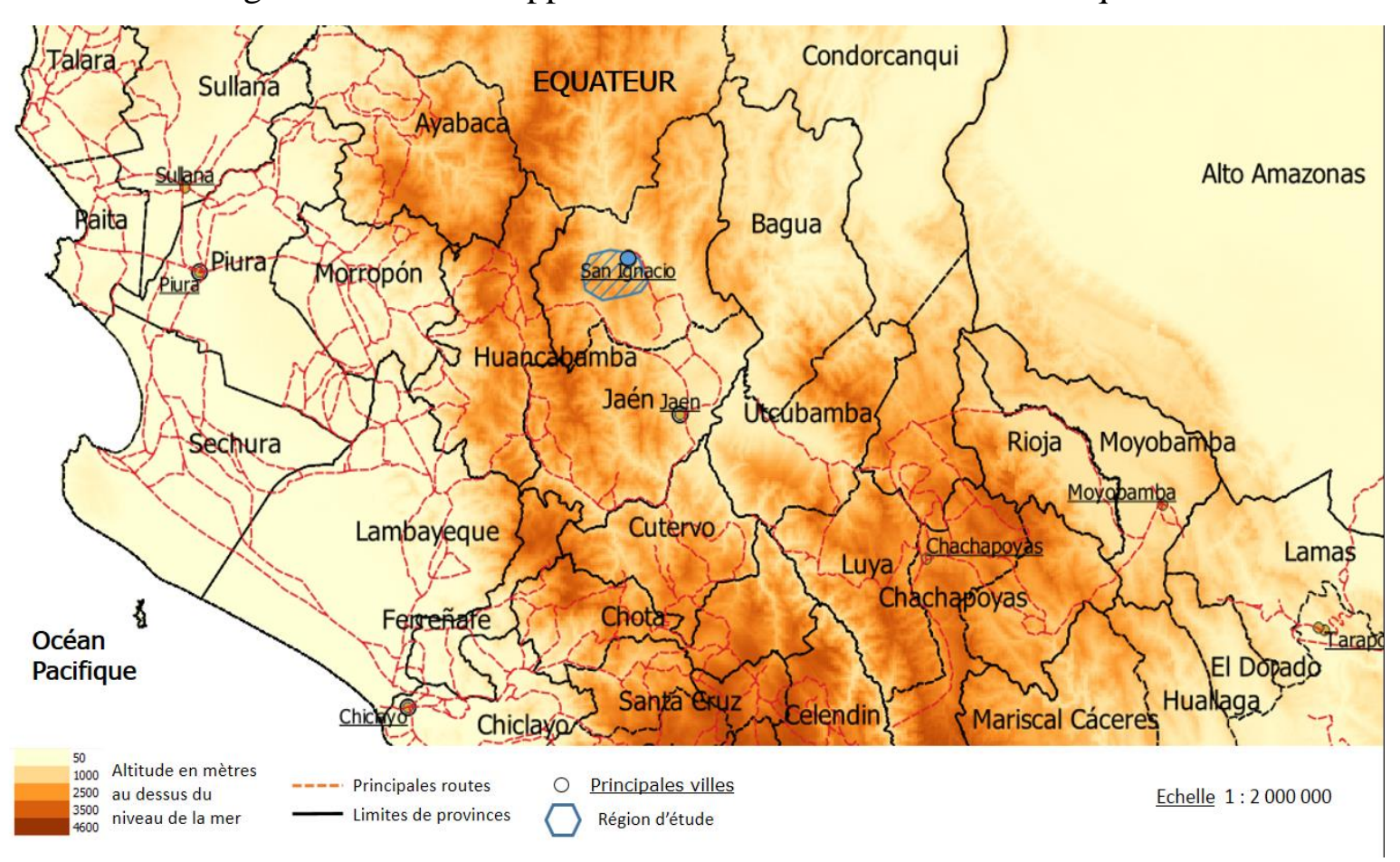

FIGURE 1. LOCALISATION DE LA REGION D’ETUDE [REALISATION : ELISE BOUËDRON ; SOURCE : OPENSTREETMAP.ORG ET GADM.ORG]

\footnotetext{
${ }^{5}$ Transformation par voie sèche : les cerises de café sont séchées au soleil avant d'être pilées pour obtenir le café vert.
} 


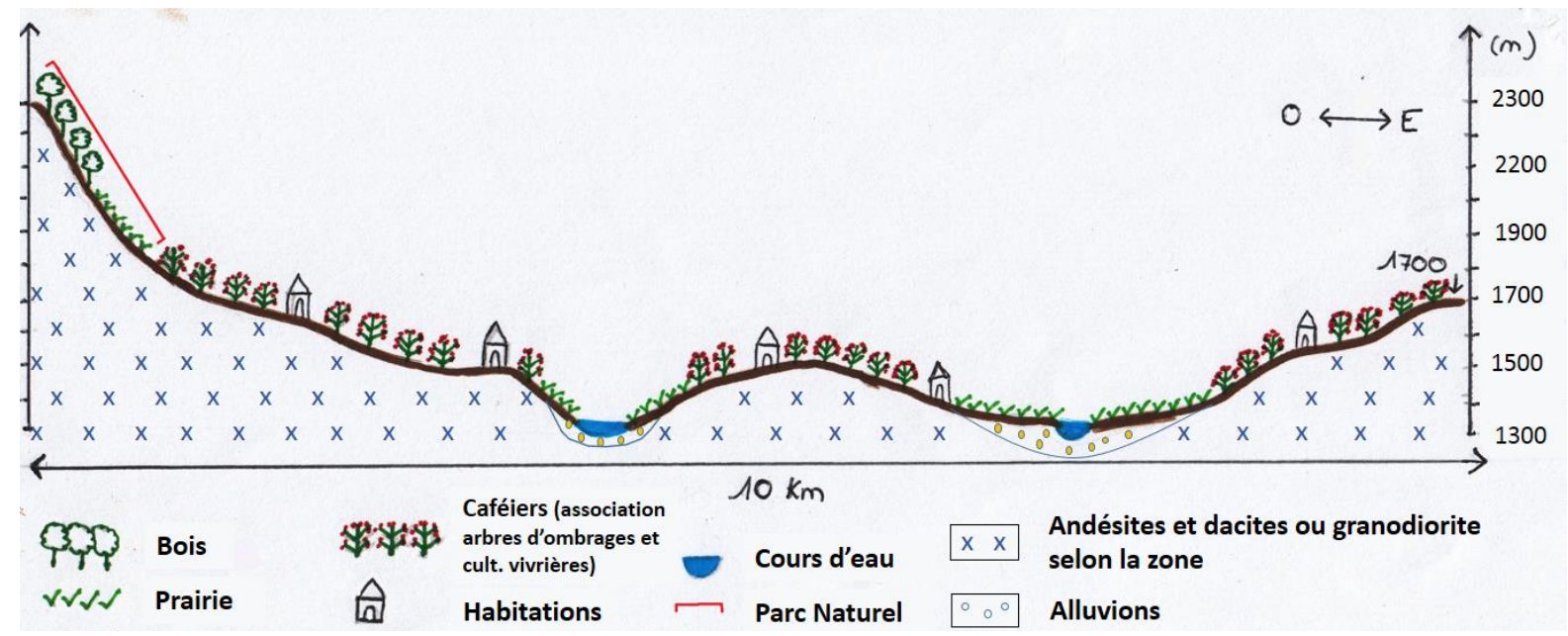

FIGURE 2. COUPE TOPOGRAPHIQUE ACTUELLE D'UNE VALLEE DE LA REGION DE SAN IGNACIO (REALISATION : AUTEUR)

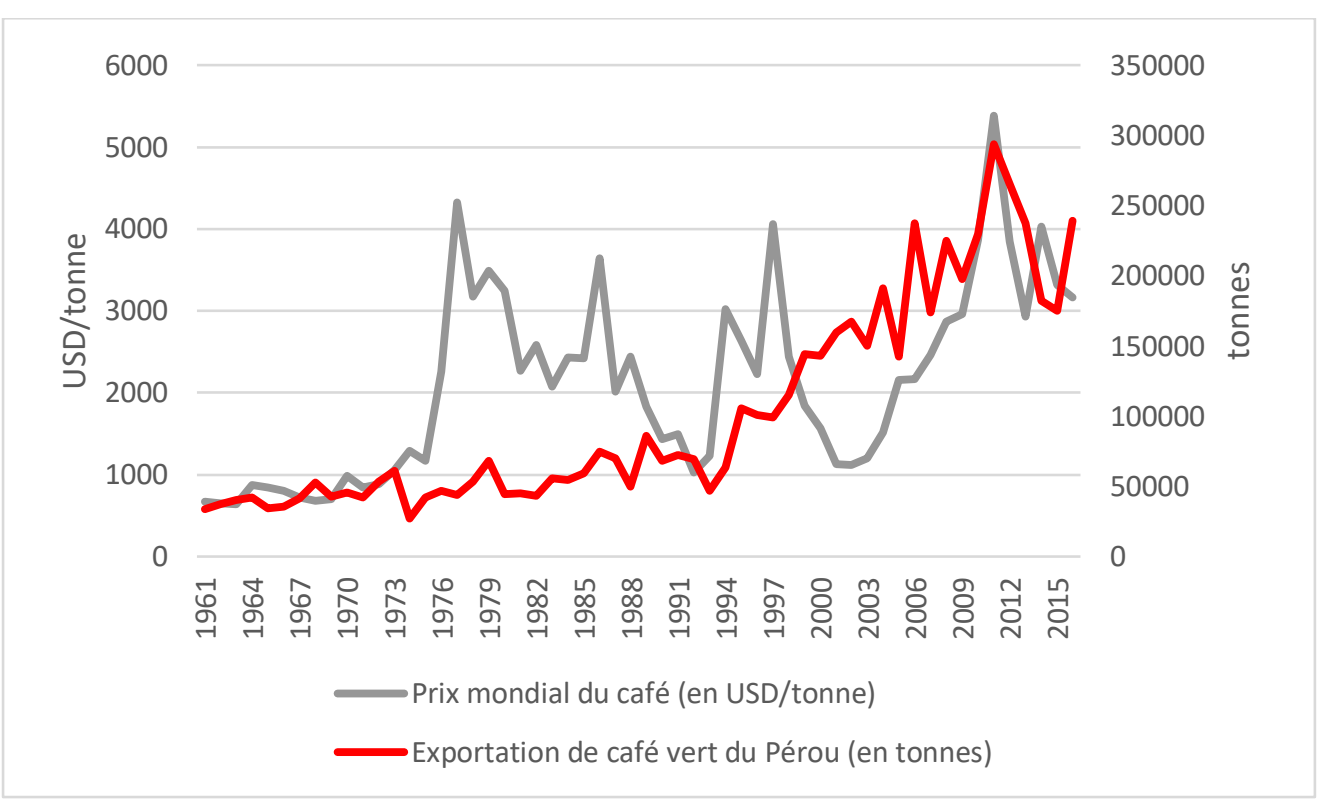

FIGURE 3. EVOLUTION DU PRIX MONDIAL GLOBAL DU CAFE ET DES EXPORTATIONS PERUVIENNES DE CAFE [SOURCE : FAO STAT]

Une spécialisation caféière encouragée par l'Etat, mais qui fragilise les plus petits producteurs

Dans les années 1970 et 1980, pour nourrir à bas coût une population urbaine grandissante, le gouvernement péruvien met en place une politique de soutien aux importations alimentaires qui rend les produits importés moins chers que les produits péruviens et qui conduit la population à remplacer en partie son alimentation traditionnelle par des denrées importées (Aubron, 2006). À partir de 1977, le cours mondial de l'arabica est multiplié par deux par rapport à la décennie précédente (figure 3). Les entretiens historiques que nous avons conduits dans la région montrent que les familles, avec une faible surface caféière (autour de 2 ha) et pas ou peu d'autres sources de revenus, convertissent alors des parcelles de cultures vivrières en café qu'elles vendront à un bon prix, pour se nourrir de produits importés (riz, pain, pâtes). C'est le début de la spécialisation caféière. Les familles qui ont un petit troupeau bovin, convertissent leurs prairies en café, car pour eux cela devient plus rentable. On observe donc une spécialisation caféière des petits producteurs qui abandonnent une part de leurs cultures vivrières ou de leur 
troupeau. Le café représente aujourd'hui 75 à $85 \%$ des revenus agricoles et plus de $95 \%$ du temps de travail agricole pour les agriculteurs qui vivent de leur seule production agricole et qui n'ont pas la possibilité de pratiquer l'élevage bovin. Ce mouvement de spécialisation impactera progressivement les autres systèmes de production.

D'autre part, alors que l'afflux de migrants se poursuit, les nouveaux arrivants peinent à accéder à suffisamment de surface pour ne vivre que de sa mise en valeur. On voit donc apparaître une classe de petits producteurs minifundistes dont le revenu dépend davantage de la vente de leur force de travail aux autres familles que de leur propre activité agricole.

En 1989 est actée la fin de l'Accord international sur le café et le prix du café chute (Prix FOB du café pour le Pérou, FAO stat.). Au même moment, les coopératives de la région 6 perdent leur principal débouché, lorsque la coopérative Nororiente, en charge de l'exportation du café de l'ensemble des coopératives du nord du Pérou, fait faillite. Enfin, pour lutter contre la crise économique et l'inflation, le président Fujimori fraichement élu applique les directives du FMI et met fin, notamment, à la Banque agraire ${ }^{7}$ qui finançait les producteurs et surtout l'activité commerciale des coopératives. Ces trois facteurs marquent la fin de l'ère des coopératives : elles s'effondrent ou bien cessent d'acheter du café. Pour les producteurs de la région d'étude, cela se traduit par une dépendance accrue vis-à-vis des acheteurs privés ${ }^{8}$ : la vente est alors contrôlée par des exportateurs privés qui sont présents depuis la collecte jusqu'à l'export (figure 4). Par ailleurs, la spécialisation caféière croissante des systèmes de production rend les producteurs plus sensibles à la variation des cours. La baisse du prix du café des années 1990 (figure 3) couplée à l'effondrement des coopératives plongent les petits et moyens producteurs dans une crise qui motivera l'intervention des bailleurs nationaux et internationaux pour la mise en place de projets de développement.

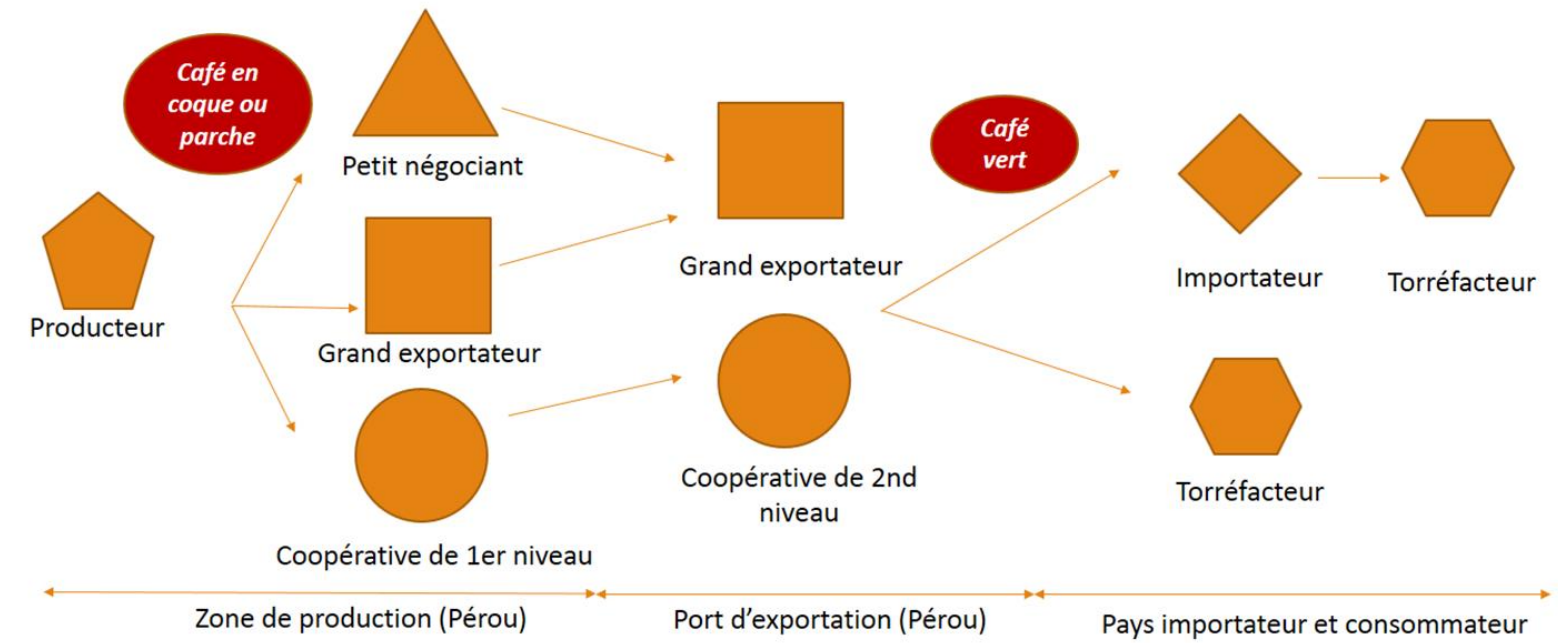

Figure 4. Filiere du cafe au Perou (realisation : Auteur)

Une orientation vers les marchés de qualité et certifiés : la recherche d'une meilleure valeur ajoutée

\footnotetext{
${ }^{6}$ À cette époque, COOPAFSI et la Casil sont parmi les coopératives les plus importantes et doivent cesser leur activité commerciale.

${ }^{7}$ Banque nationale de développement agricole : principal outil d'appui financier de l'état au secteur agricole par l'octroi de crédits.

${ }^{8}$ Parmi eux, CoInka, filiale du groupe international Neumann Kaffee Gruppe et Perhusa (Perales Huancaruna).
} 
Dans les années 1990 et 2000 s'installent dans la région de nombreux projets de développement ${ }^{9}$ pour aider les producteurs à améliorer leur situation sociale et économique dans un contexte de monopole des exportateurs privés. Ces projets veulent augmenter le rendement et la qualité du café afin d'en retirer un meilleur prix, et former à la gestion des coopératives pour encourager leur reformation sur des bases plus solides et rééquilibrer le rapport de force avec les acheteurs.

Ces projets ont apporté les ressources financières et l'expertise nécessaires à la production et à la vente à des marchés plus rémunérateurs que sont les marchés de qualité. Pour augmenter les rendements, l'itinéraire technique est modifié : installation de pépinières, plantation en ligne en suivant les courbes de niveau, optimisation de la densité de plantation, fabrication de compost pour fertiliser, gestion de l'ombrage, désherbage, etc. Le second objectif est d'améliorer la qualité du café produit pour mieux le valoriser. On encourage alors les producteurs à semer des variétés comme le Pache, le Bourbon, le Mondonovo, qui ont certes le potentiel de produire des grains de grande qualité gustative, mais sont malheureusement très sensibles aux maladies fongiques. La basse température qui règne en altitude les rend moins sensibles aux maladies qu'à moyenne (1400-1600 m) et basse (1000-1400 m) altitudes ; c'est donc à haute altitude $(1600-2000 \mathrm{~m})$ que se produira le café de meilleure qualité. La transformation par voie humide est généralisée, le café, ainsi transformé, étant réputé de meilleure qualité, il sera mieux rémunéré. Par ailleurs, la production de qualité exige davantage de soin, et donc plus de temps dans les opérations culturales et de post-récolte (environ deux fois plus de temps) : sélection pendant la récolte, la fermentation et le séchage ; fermentation longue et séchage poussé dans une région à la pluviométrie importante. Cependant, cela exige des investissements que peu de producteurs pourront faire dans un premier temps : bac de lavage, dépulpeuse en bon état et motorisée, séchoir suffisamment grand et entretenu.

À la fin des années 1990 et au début des années 2000, les coopératives réapparaissent ${ }^{10}$. Elles s'appuient sur les groupes de producteurs et sur les gestionnaires formés par les projets de développement. À partir de 2005, grâce aux trois types de certifications «commerce équitable » (Fairtrade), «biologiques » et «durables » (Rainforest Alliance, UTZ, etc.), elles gagnent de nouveaux marchés et commencent à exporter directement, sans passer par un exportateur privé. La prime au développement de 20 USD cts/livre de café vert de la certification Fairtrade a été essentielle à leur développement : toutes leurs infrastructures ont été financées en partie ou totalement par cette prime (centres de collecte, bâtiments administratifs, laboratoires d'analyse organoleptique, séchoirs collectifs, etc.) ainsi qu'une part importante des services de crédit et de formation agronomique. Aujourd'hui la quasi-totalité des coopératives ont ces trois types de certification.

C'est aussi à cette période que l'entreprise Huancaruna (aujourd'hui Perhusa, premier exportateur de café du Pérou ${ }^{11}$ ) crée son propre programme qualité en proposant à ses clients des cafés de haute qualité gustative assorti de certifications biologiques et durables. Ces programmes privés, bien que reposant sur des « associations de base » de producteurs dans chaque village, ne sont pas dirigés par les producteurs, mais ces derniers reçoivent, comme dans les coopératives, des crédits et des formations agronomiques. D'autres grands exportateurs comme Volcafé copieront ce modèle quelques années plus tard.

Les coopératives et les programmes qualité des grands exportateurs ont comme principal débouché le marché du café de qualité. C'est autour de ces deux types d'acheteurs que

\footnotetext{
${ }^{9}$ Des projets financés et gérés par l'Eglise catholique à travers l'ONG Manos Unidas, puis par l'USAID et enfin l'institution régionale Proyecto especial Jaén-Ignacio-Bagua.

${ }^{10} \mathrm{La}$ Casil et Coopafsi reprennent une activité commerciale, Cenfrocafé et Aprocassi font partie des nouvelles coopératives créées.

${ }^{11} 44000$ tonnes de café vert exportées en 2018, soit 17\% du volume total exporté par le Pérou (agrodataperu.com)
} 
s'organise localement la filière du café de qualité. Bien que les certifications justifient en ellesmêmes un prix au-dessus de celui du marché, quel que soit la qualité, les importateurs qui achètent selon ces certifications exigent une très bonne qualité (rendement physique élevé, score en tasse ${ }^{12}$ ). La majorité des producteurs possédant plus de 2 ha de café rejoignent peu à peu ces structures, attirés par un prix plus élevé.

\section{Résultats. De meilleurs revenus pour les membres des coopératives mais qui reposent en partie sur l'exclusion des plus modestes.}

\section{Des revenus plus élevés en système Fairtrade}

Nos enquêtes ont mis en évidence que depuis le développement des filières qualité dans la région, les familles intégrées au marché de la qualité (coopératives et programmes de qualité des exportateurs privés) peuvent maintenir leur revenu au-dessus du seuil de survie ${ }^{13}$ avec leur seule activité agricole (figure 5). Leur revenu journalier représente désormais 1.3 à 4 fois le salaire journalier d'un ouvrier non-qualifié dans la région, seule alternative au café pour la plupart des producteurs. Par ailleurs, les familles intégrées au système Fairtrade par l'intermédiaire d'une coopérative (environ $40 \%$ des producteurs de la région selon nos estimations) auront, en moyenne, un revenu agricole familial ${ }^{14} 15$ à $50 \%$ plus élevé que des familles pratiquant un système de production équivalent dans un programme de qualité privé d'un grand exportateur (10\% des producteurs de la région) certifié seulement «agriculture biologique » et «durable ». Cela s'explique, d'une part, par des prix plus élevés proposés au producteur (figure 6) (grâce à une meilleure répartition de la valeur ajoutée entre producteur et organisme exportateur, et grâce à la part de contrats au prix minimum Fairtrade) et, d'autre part, par de meilleures performances agronomiques en termes de rendement et de qualité (annexe 1), qui peuvent être reliées aux investissements dans les formations agronomiques financées en totalité par la prime Fairtrade.

$\mathrm{Si}$, en théorie, le prix minimum doit fonctionner comme un filet de sécurité lorsque le cours mondial chute, en pratique, il semble être d'abord lié à la capacité de la coopérative à produire des cafés spéciaux. En effet, dans la région étudiée, la totalité du café exporté a un score en tasse supérieur à 80, et une part importante des contrats Fairtrade concernent des cafés au score

\footnotetext{
${ }^{12}$ Le score en tasse mesure la qualité organoleptique du café selon une grille internationale.

Le rendement physique mesure le pourcentage de café vert exportable obtenu à partir d'un quintal de café en parche (café en parche $=$ café vert exportable + enveloppe + café avec défauts).

${ }^{13}$ Le seuil de survie est estimé en calculant les besoins annuels de base d'une famille dans la région d'étude pour l'alimentation, l'habillement, l'hygiène et l'éducation. Dans la région étudiée, les enquêtes que nous avons réalisées auprès des ménages nous permettent de l'estimer à environ 4000 euros/an, soit 2000 euros/actif dans une famille avec deux actifs.

${ }^{14}$ Le revenu agricole familial reflète le revenu économique moyen annuel d'un système de production « en vitesse de croisière ». C'est le revenu que la famille dégage annuellement en moyenne durant sa période d'activité pour vivre et accroître son capital.
} 
supérieur à $82^{15}$, dont les prix sont toujours très supérieurs au cours mondial. Les autres types de contrats (tableau 1) correspondent à des cafés biologiques, «durables » ou conventionnels, dont le montant varie selon le cours mondial. Cependant, bien que les cafés spéciaux soient hautement valorisables, y compris sans les conditions Fairtrade, la certification permet aux coopératives de se démarquer des programmes de qualité des exportateurs privés auprès des importateurs et torréfacteurs qui la valorisent auprès du consommateur. D'une part, une fraction plus importante revient au producteur, puisqu'à qualité égale, la coopérative lui reverse un prix plus élevé que l'exportateur privé. D'autre part, les 20 USD cts/livre, versés sous forme de prime Fairtrade, seront investis dans les services qui lui sont offerts par la coopérative. De plus, les coopératives de la région ont fait le choix d'une certaine solidarité entre les membres au niveau de leur politique de prix. En effet, même si la coopérative paie plus cher au producteur un café bio, «durable » ou spécial ${ }^{16}$, une part du prix des contrats Fairtrade pour les cafés spéciaux est redistribuée à tous les producteurs, indépendamment de la qualité de leur café. Les services financés par la prime Fairtrade, et donc par la vente des cafés spéciaux, profitent ainsi à tous les producteurs et participent de cette solidarité entre membres. Si les certifications biologiques ou «durables » concernent aussi les exportateurs privés (comme Huancaruna, Volcafé, CoInca, etc.) et ne permettent pas de garantir cette plus juste répartition du prix entre producteur et l'acheteur ${ }^{17}$, Fairtrade, en ne choisissant de certifier que les organisations de producteurs au sein desquelles la répartition est décidée de façon démocratique, permet aux

\footnotetext{
${ }^{15}$ Dans le cas de Cenfrocafé, la coopérative la plus importante de la région, environ la moitié des contrats Fairtrade concernent des cafés de plus de 82 points en tasse.

${ }^{16}$ En 2017, la coopérative Cenfrocafé payait un bonus de 12 USD/quintal pour café biologique ou durable, auquel se cumulait un bonus progressif selon la qualité de minimum 10 USD/quintal. 5 USD/quintal étaient versés à tous les producteurs comme redistribution du prix minimum Fairtrade, quel que soit le prix auquel avait été valorisé leur café.

${ }^{17}$ Les coopératives de la région ont des frais de fonctionnement qui varient entre 15 et 30 USD/quintal de café vert, soit entre 8 et $16 \%$ du prix minimum Fairtrade bio + prime de développement.
} 

certifications sont donc complémentaires.

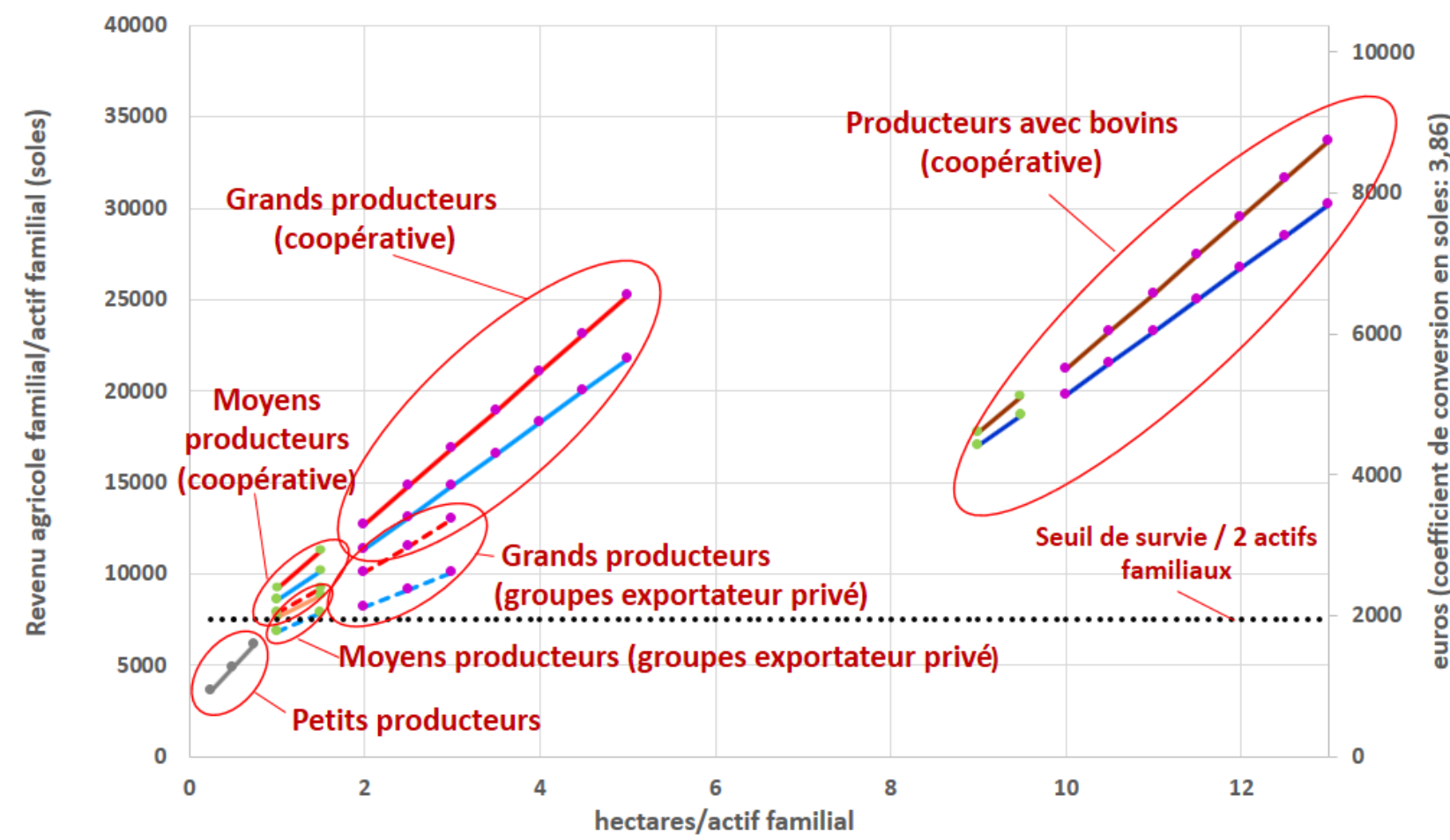

_- Grande surface, alt. moy, bov, coop

_-Grande surface, alt. moy, coop

- - Grande surface, alt. moy, programme qualité privé

_- Moyenne surface, alt. moy, bov, coop

- Moyenne surface, alt. moy, coop

---Moyenne surface, alt. moy, programme qualité privé

- Moyenne surface, alt. haute, programme qualité privé

_-Grande surface, alt. haute, bov, coop

$\longrightarrow$ Grande surface, alt. haute, coop

- - Grande surface, alt. haute, programme qualité privé

- Moyenne surface, alt. haute, bov, coop

_-Moyenne surface, alt. haute, coop

—-Moyenne surface, alt. basse, coop

_._Faible surface, toutes alt., filière standard

FIGURE 5. REVENU AGRICOLE FAMILIAL PAR ACTIF FAMILIAL EN FONCTION DE LA SURFACE TOTALE PAR ACTIF FAMILIAL (PRAIRIES INCLUSES), (ALT. : ALTITUDE ; BOV : ElEVAGE BOVIN ; COOP : COOPERATIVE) [REALISATION : AUTEUR A PARTIR DE DONNEES D'ENQUETES]

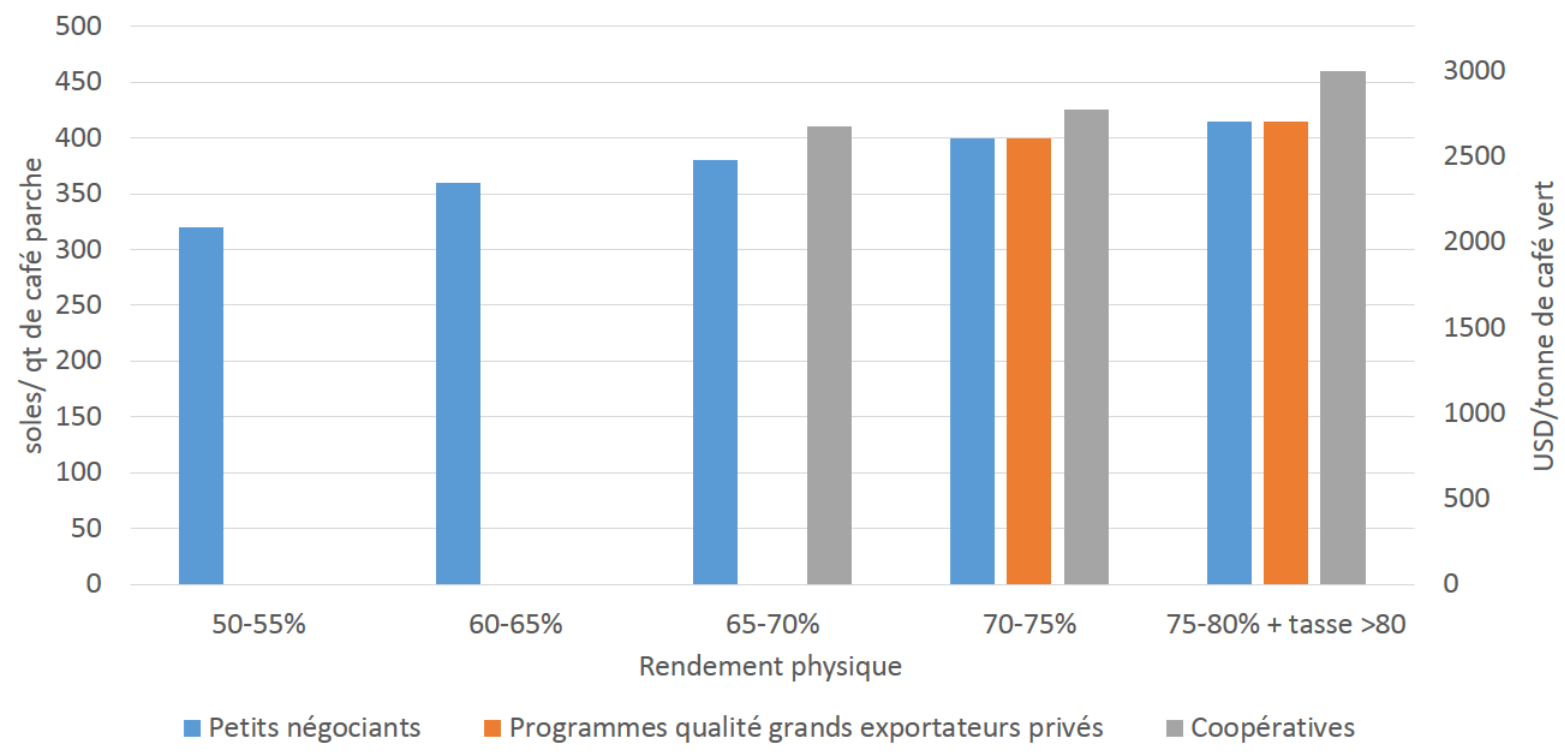

Figure 6. Prix moyen du Cafe paye au producteur, Selon le Rendement Physique et le debouche, entre 2012 et 2017 [Source : enquetes + Agence Agraire de la Province de San Ignacio] 
TABLEAU 1. TYPES DE CONTRATS ENTRE COOPERATIVES ET IMPORTATEUR/TORREFACTEUR (UN CAFE FT OU BIO PEUT TRES

BIEN ETRE VENDU COMME CONVENTIONNEL SI PEU DE DEMANDE).

\begin{tabular}{|c|c|c|}
\hline Fairtrade & Bio ou « durable » & Conventionnel \\
\hline $\begin{array}{l}\text { Prix : En théorie égal ou supérieur } \\
\text { au «prix minimum » additionné de } \\
\text { la «prime de développement } \\
\text { Dans les faits, il lui est rarement } \\
\text { supérieur, il est donc stable et égal } \\
\text { au «prix minimum + prime au } \\
\text { développement»; }\end{array}$ & $\begin{array}{l}\text { Multitude de labels. } \\
\text { Prix généralement supérieur au } \\
\text { cours de l'arabica mais pas fixé } \\
\text { dans le cahier des charges, il varie } \\
\text { avec le cours. }\end{array}$ & $\begin{array}{l}\text { Prix qui varie selon la qualité en } \\
\text { tasse. Prix moyen égale le prix des } \\
\text { contrats « bio ou durables » grâce } \\
\text { aux contrats pour des cafés de très } \\
\text { haute qualité (> } 83 \text { en tasse). }\end{array}$ \\
\hline $\begin{array}{l}\text { Bonus de } 30 \text { USD cts/livre si } \\
\text { contrat en bio en plus du FT. } \\
\text { Concerne la quasi-totalité des cafés } \\
\text { spéciaux avec une tasse supérieure } \\
\text { à } 82 \text {. }\end{array}$ & & \\
\hline
\end{tabular}

Les enquêtes détaillées permettent cependant de mettre en évidence que la certification Fairtrade ne permet pas de gommer les inégalités préexistantes d'accès aux ressources. Les producteurs possédant de larges surfaces en café ou un élevage bovin ont des revenus bien plus élevés que les autres membres des coopératives. Dans le cadre de ce travail de recherche, nous avons élaboré une typologie des producteurs qui tient compte, notamment, de leur surface en café : on parlera de «petits », de «moyens » et de «grands » producteurs (annexe 1, respectivement environ $40 \%, 50 \%$ et $10 \%$ des producteurs de la région), seules les deux dernières catégories sont membres des coopératives. Les standards du Fairtrade exigent d'une coopérative certifiée qu'elle ait au moins $50 \%$ de « petits producteurs » selon une définition qui diffère de celle que nous proposons, adaptée spécifiquement à la région de San Ignacio. Pour Fairtrade, un petit producteur «ne dépend pas structurellement d'une main-d'œuvre salariée permanente, et gère son exploitation essentiellement par sa propre main-d'œuvre et celle de sa famille. Il peut engager des travailleurs si le travail fourni par sa famille n'est pas suffisant pendant les saisons hautes, pour les semailles et la récolte. Cependant, les travailleurs ne sont habituellement pas employés de façon permanente pendant toute l'année de production de ces cultures » (FTO, 2011). Les petits et moyens producteurs de notre classification répondent bien à cette exigence. En revanche, les «grands producteurs », qui représentent une part non négligeable des membres des coopératives, sont bien dépendants d'une main-d'œuvre salariée toute l'année. Bien que ces derniers représentent environ $25 \%$ des membres des coopératives et que les standards Fairtrade soient donc respectés, on peut interroger le fait qu'ils bénéficient - au même titre que les autres - des conditions Fairtrade alors que l'on s'éloigne de la petite agriculture familiale avec des travaux agricoles réalisés à plus de 50\% par une main-d'œuvre salariée contre 20 à $30 \%$ chez les producteurs moyens. Le revenu d'un grand producteur peut représenter jusqu'à 3 fois le revenu d'un autre membre de la coopérative, et s'il combine le café à un élevage bovin, jusqu'à 4.5 fois le seuil de survie. D'après nos estimations, ces grands producteurs représentent à peu près $10 \%$ des producteurs de la région.

\subsection{L'exclusion des plus petits producteurs}

Si la superficie moyenne de café des membres des coopératives oscille autour de 3 ha par famille de deux actifs familiaux, à l'échelle de la région elle tombe à 1.5 ha (Agence agraire de la Province de San Ignacio). Les plus petits producteurs ne sont donc pas intégrés aux 
coopératives, et de façon générale, aux marchés de qualité. Une des principales raisons en est que la transition vers une production de café de qualité exige des investissements (au minimum 2200 euros pour une exploitation moyenne de 2 à 3 ha, soit un peu plus de la moitié du seuil

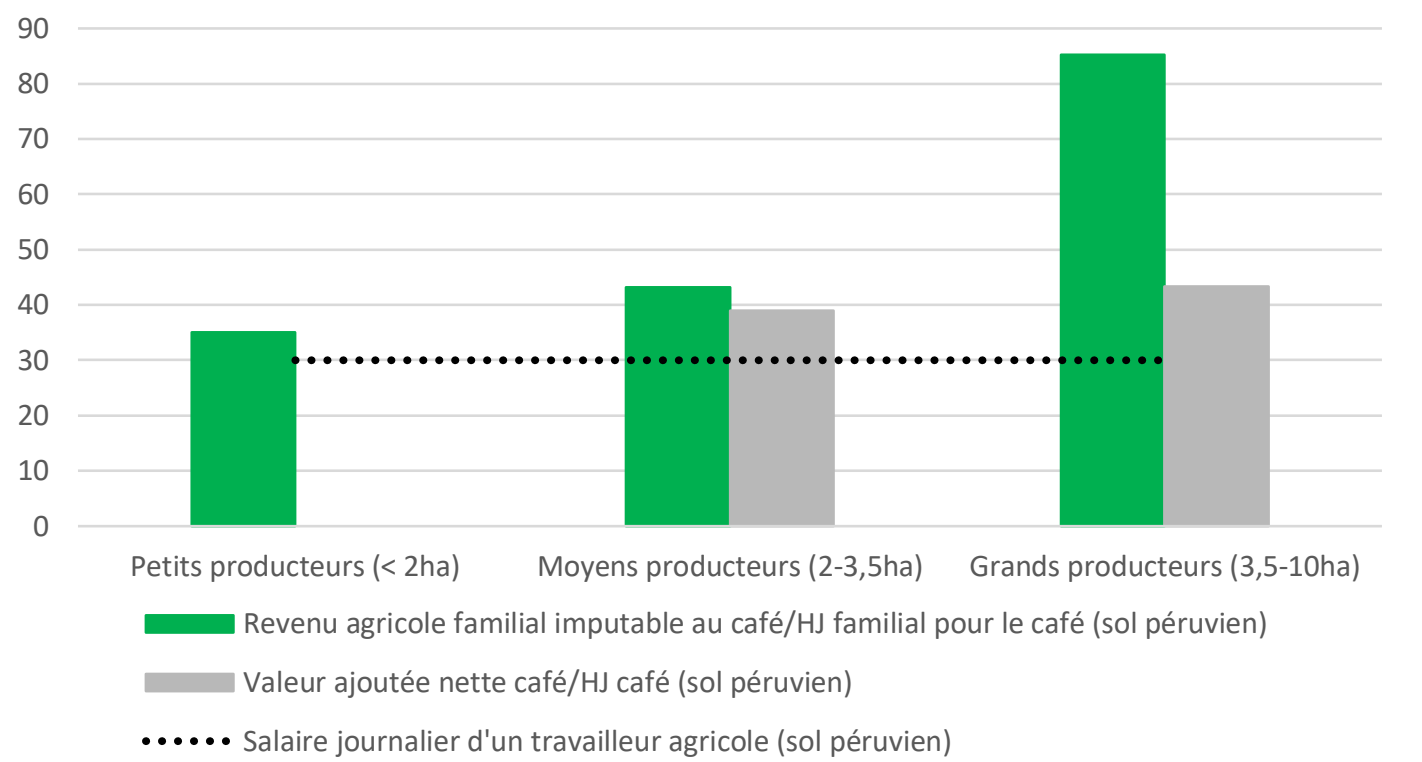

FIgURE 7. COMPARAISON DE LA REMUNERATION JOURNALIERE DES ACTIFS FAMILIAUX, DES SALARIES ET DE LA PRODUCTIVITE DU travail journaliere (HJ : Journee de travail ; VAN : Valeur Ajoutee NetTe ; Prog : Programme Qualite EXPORTATEUR PRIVE) [REALISATION : AUTEUR]

annuel de survie). Or, même si les coopératives et les programmes de qualité privés soutiennent leurs membres à travers des crédits, ils ne financent pas la totalité de l'investissement. Sont donc exclus les plus petits producteurs, notamment ceux dont la surface caféière est insuffisante (moins de 2 ha) pour assurer leur revenu avec le seul café et dont l'activité économique secondaire permet à peine d'atteindre le seuil de survie. Ces producteurs ont adopté une partie des techniques apportées par les projets de développement (par exemple les pépinières, la transplantation en ligne, le désherbage, etc.), mais pas celles qui demandent des investissements importants, comme le séchoir ou le bac de lavage. Ils n'achètent pas de fertilisants et ne cherchent pas à semer des variétés à haut potentiel de qualité qu'ils ne pourront pas valoriser, faute de matériel de post-récolte assez performant. Le café est alors fermenté dans des sacs en plastique et séché à même le sol, sans protection contre les intempéries. Le café produit n'est donc valorisable que sur le marché standard, où les prix sont peu rémunérateurs (figures 5 et 6). Ils n'ont pas accès au crédit ou au conseil agronomique. Ces petits producteurs devront travailler chez les autres producteurs pour compléter leurs revenus pour une rémunération journalière équivalente au salaire minimum péruvien. Dans les plus grandes exploitations caféières, celles qui font largement appel à la force de travail salariée, on constate que le revenu dégagé par journée de travail familial est largement supérieur à la rémunération journalière des salariés (figure 7). Ces petits producteurs, vendeurs de force de travail, représentent presque la moitié des familles de la région. Ils sont les plus nombreux, et les plus disponibles puisque leur faible surface leur demande peu de travail.

\subsection{Fairtrade ne suffit pas à atténuer les risques liés à la spécialisation}

Si un des objectifs de Fairtrade est de stabiliser les revenus des producteurs, l'étude a montré que ce n'est pas le cas pour la région de San Ignacio. Des négociations abusives de la part des acteurs situés en aval de la filière seraient en cause. Certains d'entre eux réclameraient des 
réductions sur les lots non Fairtrade en échange de l'obtention de contrats Fairtrade ${ }^{18}$, entamant alors l'augmentation potentielle du prix au producteur lorsque la part de contrats Fairtrade est élevée. De plus, en période de prix bas (c'est-à-dire très inférieurs au prix minimum Fairtrade de 190 USD cents/livre pour un café arabica lavé bio), les importateurs préféreraient acheter du café non certifié. Cela expliquerait que le prix payé au producteur, même associé à une coopérative certifiée, suive plus ou moins les variations du cours mondial de l'arabica (ce qui a été confirmé par les enquêtes). Dans un système qui dépend autant des cours du café, les producteurs sont fragilisés. En 2018, certains producteurs, même intégrés au Fairtrade, ont vu leurs revenus baisser et se rapprocher dangereusement du seuil de survie. La spécialisation très poussée pose alors problème, puisque le revenu de la plupart des familles en est extrêmement dépendant. Elle les soumet d'autant aux aléas du marché.

Le café de la région est cultivé au sein d'un système agroforestier à ombrage faible à moyen selon l'altitude, ce qui est, en termes de biodiversité et de protection de la fertilité des sols, plus performant qu'un système en plein soleil comme il peut être courant dans d'autres régions. Cependant, il est courant qu'une variété de café prédomine largement dans une parcelle. Les caféiers seraient alors plus sensibles aux attaques de nuisibles, phénomène aggravé par le changement climatique (Toniutti et al., 2017). Dès lors, la course aux variétés résistantes (les nuisibles s'adaptent, le recours à une nouvelle variété est nécessaire régulièrement) semble privilégiée par rapport à une réflexion de fond qui rechercherait l'adaptation du système de production dans son ensemble. En outre, bien que les producteurs pratiquent en majorité une agriculture biologique, ils sont dépendants de l'usage de fertilisants pour assurer le niveau de production minimal exigé par certaines coopératives pour amortir les coûts de service au producteur. Et, par ailleurs, les fertilisants autorisés en agriculture biologiques sont deux fois plus chers qu'en agriculture conventionnelle qui, de fait, constitue leur principal poste de dépense $^{19}$. Enfin, si l'action combinée des fertilisants achetés et de l'adoption de variétés à haut rendement a permis de doubler les rendements à l'échelle de la région depuis 2000 (statistiques de Agence agraire de la Province de San Ignacio et de nos enquêtes), cela signifie aussi un travail de récolte beaucoup plus important ${ }^{20}$. Les producteurs sont donc très dépendants de la main-d'œuvre extérieure à la famille, qui est aujourd'hui principalement salariée et issue de la classe des petits producteurs exclus des filières de qualité. Bien que le niveau de rémunération soit bas (figure 7), cela reste un poste de dépense important pour les producteurs.

\section{Conclusion}

Dans la région de San Ignacio, les agriculteurs ont tiré parti des conditions agroclimatiques favorables et d'une demande mondiale en expansion pour faire du café une source de revenus complémentaire à l'agriculture vivrière. Cependant la politique menée par le gouvernement dans les années 1970 et 1980 a encouragé la spécialisation caféière tout en rendant les plus petits producteurs plus vulnérables à la variation des cours. Aujourd'hui, le marché du café de qualité et certifié « biologique » et «durable » permet aux producteurs - qui ont pu réaliser les investissements nécessaires - de mieux valoriser leur café et de vivre de cette

\footnotetext{
${ }^{18}$ L'ensemble de la production d'une coopérative certifiée Fairtrade est certifiée, mais la demande actuelle de café certifié ne permet pas d'absorber toute la production ; une part plus ou moins importante (30 à $70 \%$ de la production) doit être vendue hors conditions Fairtrade.

${ }^{19}$ Pour une production moyenne de 20 quintaux/ha à l'échelle de la région : 1400 soles/ha pour le bio et 700 soles/ha pour le conventionnel. Pour un producteur moyen en bio cela représente la moitié des coûts de production du café (consommations intermédiaires + salaire de la main d'œuvre non familiale).

${ }^{20}$ Pour récolter 20 qt (la production moyenne par hectare), il faut environ $80 \mathrm{HJ}$.
} 
seule activité. Le revenu journalier représente désormais 1.3 à 4 fois le salaire journalier d'un ouvrier non qualifié dans la région, seule alternative au café pour la plupart des producteurs. Cependant, les certifications organiques et durables ne garantissent pas une décision démocratique quant à la répartition du prix entre producteur et organisme collecteur. Fairtrade, en ne certifiant que les organisations de producteurs, permet aux producteurs des coopératives de la région de mieux profiter des prix élevés offerts pour les autres certifications tout en leur assurant de se démarquer de leurs concurrents privés. De plus, par sa prime elle soutient la pérennité et le développement des organisations de producteurs en finançant une large part de leurs investissements et de leurs services au producteur. Grâce au travail des coopératives, le revenu des producteurs certifiés Fairtrade est aujourd'hui 15 à 50\% supérieur à celui des familles accédant à des ressources comparables et produisant un café de qualité équivalente, dans des filières non certifiées Fairtrade. Cependant, si Fairtrade a participé à une réelle amélioration des conditions de vie des producteurs qui ont été intégrés à ces filières, les investissements nécessaires pour la production de qualité excluent des filières qualité près de la moitié des producteurs de la région. Paradoxalement, ce sont ces exclus de la filière qui constituent la main-d'œuvre bon marché sur laquelle reposent les filières de qualité, notamment celles certifiées Fairtrade, biologique et/ou « durable». Pour leurs membres, les certifications ne suffisent pas à contrer les effets de la spécialisation, les producteurs sont dépendants d'une main-d'œuvre, elle-même paupérisée, et de l'achat d'intrants à des prix élevés, et ils restent sensibles aux fluctuations des cours, malgré le filet de sécurité que devrait jouer le prix minimum.

Pour recentrer les avantages du système Fairtrade sur les petits producteurs, il conviendrait de favoriser l'intégration des plus petits aux coopératives et de diminuer les avantages du Fairtrade pour les grands. On pourrait envisager la construction d'infrastructures de transformation postrécolte mise en commun pour les membres des coopératives. D'autre part, on pourrait imaginer limiter d'avantage la quantité vendue par chaque producteur aux avantages Fairtrade, afin d'accroître le nombre de bénéficiaires potentiels. Les nouveaux standards de 2019 (FLO, 2019) vont dans ce sens, en passant de 50 à $66 \%$ des volumes vendus aux conditions Fairtrade, la production qui doit provenir des petits producteurs.

La diminution des revenus issus du café couplé à la pénibilité du travail, pousse de plus en plus les jeunes à s'orienter vers d'autres activités économiques (et à quitter la région). La province a aujourd'hui un solde migratoire négatif. Cela pose la question de la pérennité des exploitations caféières de la région, y compris celles des organisations certifiées Fairtrade. Pour lutter contre la sensibilité au variations des cours de l'arabica, la dépendance à une main-d'œuvre salariée paupérisée et le recours à des intrants externes coûteux pour maintenir les rendements, il pourrait être envisagé, à long terme, de revenir à des systèmes plus diversifiés (association avec des céréales et des légumineuses par exemple) avec moins de caféiers, et donc moins dépendants de la main-d'œuvre extérieure pour alléger les charges des ménages. Introduire de nouvelles cultures au sein du café et développer les activités d'élevage, pourraient permettre de diversifier les sources de revenus et donc de les stabiliser, d'entretenir/d'améliorer la fertilité des sols, pour une dépendance moindre aux engrais achetés. Il serait intéressant pour les coopératives et le mouvement Fairtrade de réaliser des analyses fines de filières, pour ensuite soutenir chez les producteurs la mise en place d'une ou plusieurs activités agricoles compatibles avec les conditions agroécologiques de la région, complémentaires du café, et pour lesquelles il existe un marché. 
AGENCE AGRAIRE DE LA PROVINCE DE SAN IGNACIO, statistiques agricoles (données brutes), disponible à l'agence.

Aubron, C. (2006). 'Le lait des Andes vaut-il de l'or ? Logiques paysannes et insertion marchande de la production fromagère andine', thèse de doctorat en agriculture comparée, sous

la direction d'Hubert Cochet, Institut National Agronomique Paris-Grignon, 549p.

Bureau d'Analyse Sociétale pour une Information Citoyenne (2018) 'Café : la success story qui cache la crise, Etude sur la durabilité de la filière café', Commerce Equitable France, Biocoop, Ethiquable, Ecocert, Bio Partenaire, Basic, 100p.

Bouëdron E.(2018). ' Diagnostic agraire d'une région de piémont productrice de café au Pérou, mémoire de fin d'études en agriculture comparée', sous la direction d'Hubert Cochet, AgroParisTech, 2018, 93p.

Cochet H. et Devienne S. (2006). 'Fonctionnement et performances économiques des systèmes de production agricole : une démarche à l'échelle régionale', Cahiers Agricultures vol. 15, $\mathrm{n}^{\circ}$ 6, novembre-décembre 2006, 578-583p.

Cochet H., Devienne S. et Dufumier M. (2007). 'L'agriculture comparée : une discipline de synthèse ?', Economie rurale, 297-298/janvier-mars 2007 :99-110.

Cochet, H. (2011). 'L'Agriculture Comparée', QUAE/NSS-Dialogues, coll Indisciplines, 159 p.

Diaz Pedregal, V. (2006). 'Commerce équitable et organisations de producteurs. Le cas des caféiculteurs andins au Pérou, en Equateur et en Bolivie', Paris, L'Harmattan, 288p.

Eberhart, N. (2007). 'Dispositif de mesure d'impact du commerce équitable sur les organisations et familles paysannes et leurs territoires. Proposition méthodologique', AVSFLyon, 120p.

FAIRTRADE INTERNATIONAL (2011). 'Standard du Commerce Equitable Fairtrade pour les organisations de petits producteurs', www.fairtrade.net/fileadmin/user_upload/content/2009/standards/SPO_FR_v1.5.pdf

FAIRTRADE INTERNATIONAL (2019). 'Standard du Commerce Equitable Fairtrade pour les organisations de petits producteurs', www.fairtrade.net/fileadmin/user_upload/content/2009/standards/SPO_FR.pdf

FAIRTRADE INTERNATIONAL (2017). 'Monitoring the Scope', 58p. www.fairtrade.net/fileadmin/user_upload/content/FairtradeMonitoringReport_9thEdition_lores.pdf

FAOSTAT, Food and Agriculture Organization. 'Café vert exporté par le Pérou en valeur et en tonnage, 1970-2017' (données brutes), disponible en ligne sur: http://www.fao.org/faostat/en/\#data/TP 
Mendez, V. E., Bacon, C. M., Olson, M., Petchers, S., Herrador, D., Carranza, C., et al. (2010). Effects of Fair Trade and organic certifications on small-scale coffee farmer households in Central America and Mexico. Renewable Agriculture and Food Systems, 25(3), 236-251.

Nelson, V. (ed.) (2017), 'Fairtrade Impacts: Lessons from around the world' Rugby, UK: Practical Action Publishing <http://dx.doi.org/10.3362/9781780449067>

Oya, C., F. Schaefer and D. Skalidou (2018), 'The effectiveness of agricultural certification in developing countries: A systematic review.' World Development 112(112): 282-312.

Ruben, R. et Fort, R. (2011). 'The impact of fairtrade certification for coffee farmers in Peru', World Development. Volume 40 no. 3; pp 570-582.

Ruben, R. et Zuniga, G. (2011). 'How standards compete: comparative impact of coffee certification schemes in Northern Nicaragua', Supply Chain Management, 16/2, pp. 98-109.

Toniutti L, Breitler J-C, Etienne H, Campa C, Doulbeau S, Urban L, Lambot C, Pinilla J-CH et Bertrand B (2017). 'Influence of Environmental Conditions and Genetic Background of Arabica Coffee (C. arabica L) on Leaf Rust (Hemileia vastatrix) Pathogenesis', Front. Plant Sci. 8:2025.

Valkila, J., \& Nygren, A. (2009). 'Impacts of fair trade certification on coffee farmers, cooperatives, and laborers in Nicaragua', Agriculture and Human Values, 27(3), 321-333. 


\begin{tabular}{|c|c|c|c|c|}
\hline $\begin{array}{l}\text { Système de } \\
\text { production }\end{array}$ & $\begin{array}{l}\text { Surface en café } \\
\text { et } \% \text { de main } \\
\text { d'œuvre salariée }\end{array}$ & $\begin{array}{l}\text { Altitude, rendement } \\
\text { et qualité du café }\end{array}$ & Equipement & $\begin{array}{l}\text { Activité } \\
\text { économique } \\
\text { complémentaire }\end{array}$ \\
\hline $\begin{array}{l}\text { Petits producteurs } \\
\text { en filière standard }\end{array}$ & $\begin{array}{l}<2 \text { ha } \\
\text { Pas de salariés }\end{array}$ & $\begin{array}{l}\text { Toutes altitudes } \\
740-1010 \mathrm{~kg} \text { cv/ha } \\
\text { Qualité basse à } \\
\text { standard }\end{array}$ & $\begin{array}{l}\text { Dépulpeuse } \\
\text { manuelle }\end{array}$ & $\begin{array}{l}\text { Salarié agricole } \\
\text { dans les autres } \\
\text { systèmes }\end{array}$ \\
\hline $\begin{array}{l}\text { Moyens } \\
\text { producteurs, alt. } \\
\text { haute, coop. }\end{array}$ & $\begin{array}{l}2-3,5 \text { ha } \\
20-30 \%\end{array}$ & $\begin{array}{l}1600-2000 \mathrm{~m} \text { (alt. h.) } \\
920-1010 \mathrm{~kg} \mathrm{cv} / \mathrm{ha} \\
\text { Qualité excellente }\end{array}$ & $\begin{array}{l}\text { Dépulpeuse } \\
\text { motorisée, bac de } \\
\text { lavage, séchoir }\end{array}$ & $\begin{array}{l}\text { Parfois élevage } \\
\text { bovin si accès à } \\
\text { la terre }\end{array}$ \\
\hline $\begin{array}{l}\text { Moyens } \\
\text { producteurs, alt. } \\
\text { haute, prog. }\end{array}$ & $\begin{array}{l}2-3,5 \text { ha } \\
20-30 \%\end{array}$ & $\begin{array}{l}1600-2000 \mathrm{~m} \\
830-920 \mathrm{~kg} \mathrm{cv} / \mathrm{ha} \\
\text { Qualité excellente }\end{array}$ & $\begin{array}{l}\text { Dépulpeuse } \\
\text { motorisée, bac de } \\
\text { lavage, séchoir }\end{array}$ & \\
\hline $\begin{array}{l}\text { Moyens } \\
\text { producteurs, alt. } \\
\text { moyenne, coop. }\end{array}$ & $\begin{array}{l}2-3,5 \text { ha } \\
20-30 \%\end{array}$ & $\begin{array}{l}1400-1600 \mathrm{~m} \text { (alt. m.) } \\
1150-1240 \mathrm{~kg} \mathrm{cv} / \mathrm{ha} \\
\text { Qualité bonne }\end{array}$ & $\begin{array}{l}\text { Dépulpeuse } \\
\text { motorisée, bac de } \\
\text { lavage, séchoir }\end{array}$ & $\begin{array}{l}\text { Parfois élevage } \\
\text { bovin si accès à } \\
\text { la terre }\end{array}$ \\
\hline $\begin{array}{l}\text { Moyens } \\
\text { producteurs, alt. } \\
\text { moyenne, prog. }\end{array}$ & $\begin{array}{l}2-3,5 \text { ha } \\
20-30 \%\end{array}$ & $\begin{array}{l}1400-1600 \mathrm{~m} \\
1060-1150 \mathrm{~kg} \text { cv/ha } \\
\text { Qualité bonne }\end{array}$ & $\begin{array}{l}\text { Dépulpeuse } \\
\text { motorisée, bac de } \\
\text { lavage, séchoir }\end{array}$ & \\
\hline $\begin{array}{l}\text { Moyens } \\
\text { producteurs, alt. } \\
\text { basse, coop. }\end{array}$ & $\begin{array}{l}2-3,5 \text { ha } \\
20-30 \%\end{array}$ & $\begin{array}{l}\text { 1000-1400m (alt. b.) } \\
\text { 1060-1150 kg cv/ha } \\
\text { Qualité standard à } \\
\text { bonne }\end{array}$ & $\begin{array}{l}\text { Dépulpeuse } \\
\text { motorisée, bac de } \\
\text { lavage, séchoir }\end{array}$ & \\
\hline $\begin{array}{l}\text { Grands } \\
\text { producteurs, alt. } \\
\text { haute, coop. }\end{array}$ & $\begin{array}{l}3,5-10 \text { ha } \\
>50 \%\end{array}$ & $\begin{array}{l}1600-2000 \mathrm{~m} \\
920-1010 \mathrm{~kg} \text { cv/ha } \\
\text { Qualité excellente }\end{array}$ & $\begin{array}{l}\text { Dépulpeuse } \\
\text { motorisée, bac de } \\
\text { lavage, séchoir }\end{array}$ & $\begin{array}{l}\text { Parfois élevage } \\
\text { bovin si accès à } \\
\text { la terre }\end{array}$ \\
\hline $\begin{array}{l}\text { Grands } \\
\text { producteurs, alt. } \\
\text { haute, prog. }\end{array}$ & $\begin{array}{l}3,5-10 \text { ha } \\
>50 \%\end{array}$ & $\begin{array}{l}1600-2000 \mathrm{~m} \\
830-920 \mathrm{~kg} \mathrm{cv} / \mathrm{ha} \\
\text { Qualité excellente }\end{array}$ & $\begin{array}{l}\text { Dépulpeuse } \\
\text { motorisée, bac de } \\
\text { lavage, séchoir }\end{array}$ & \\
\hline $\begin{array}{l}\text { Grands } \\
\text { producteurs, alt. } \\
\text { moyenne, coop. }\end{array}$ & $\begin{array}{l}3,5-10 \text { ha } \\
>50 \%\end{array}$ & $\begin{array}{l}1400-1600 \mathrm{~m} \\
1150-1240 \mathrm{~kg} \mathrm{cv} / \mathrm{ha} \\
\text { Qualité bonne }\end{array}$ & $\begin{array}{l}\text { Dépulpeuse } \\
\text { motorisée, bac de } \\
\text { lavage, séchoir }\end{array}$ & $\begin{array}{l}\text { Parfois élevage } \\
\text { bovin si accès à } \\
\text { la terre }\end{array}$ \\
\hline $\begin{array}{l}\text { Grands } \\
\text { producteurs, alt. } \\
\text { moyenne, prog. }\end{array}$ & $\begin{array}{l}3,5-10 \text { ha } \\
>50 \%\end{array}$ & $\begin{array}{l}1400-1600 \mathrm{~m} \\
1060-1150 \mathrm{~kg} \mathrm{cv} / \mathrm{ha} \\
\text { Qualité bonne }\end{array}$ & $\begin{array}{l}\text { Dépulpeuse } \\
\text { motorisée, bac de } \\
\text { lavage, séchoir }\end{array}$ & \\
\hline
\end{tabular}
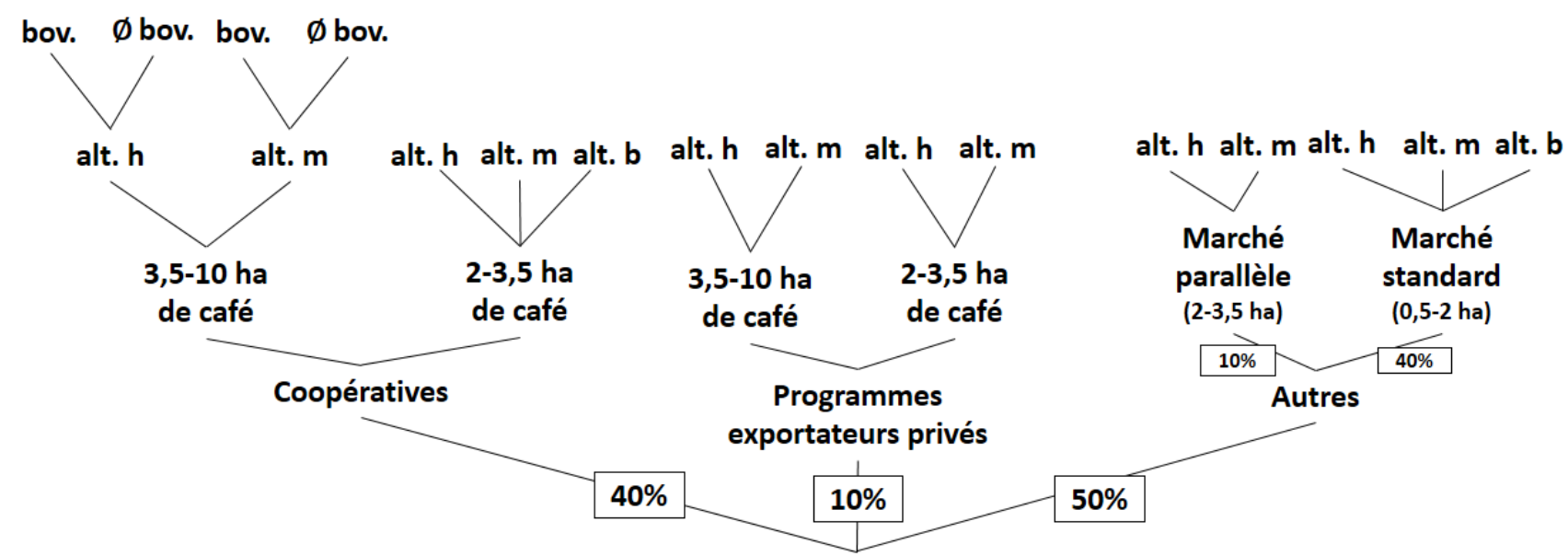

ANNEXE 1. TYPOLOGIE, PRINCIPALES CARACTERISTIQUES ET PROPORTIONS RELATIVES DES SYSTEMES DE PRODUCTION DE LA REGION DE SAN

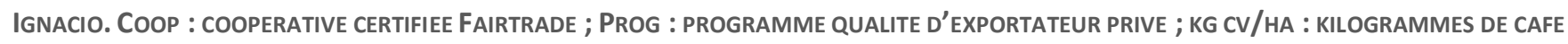

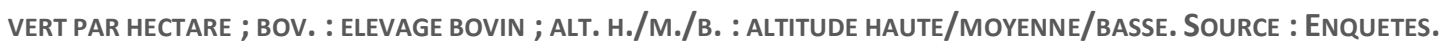




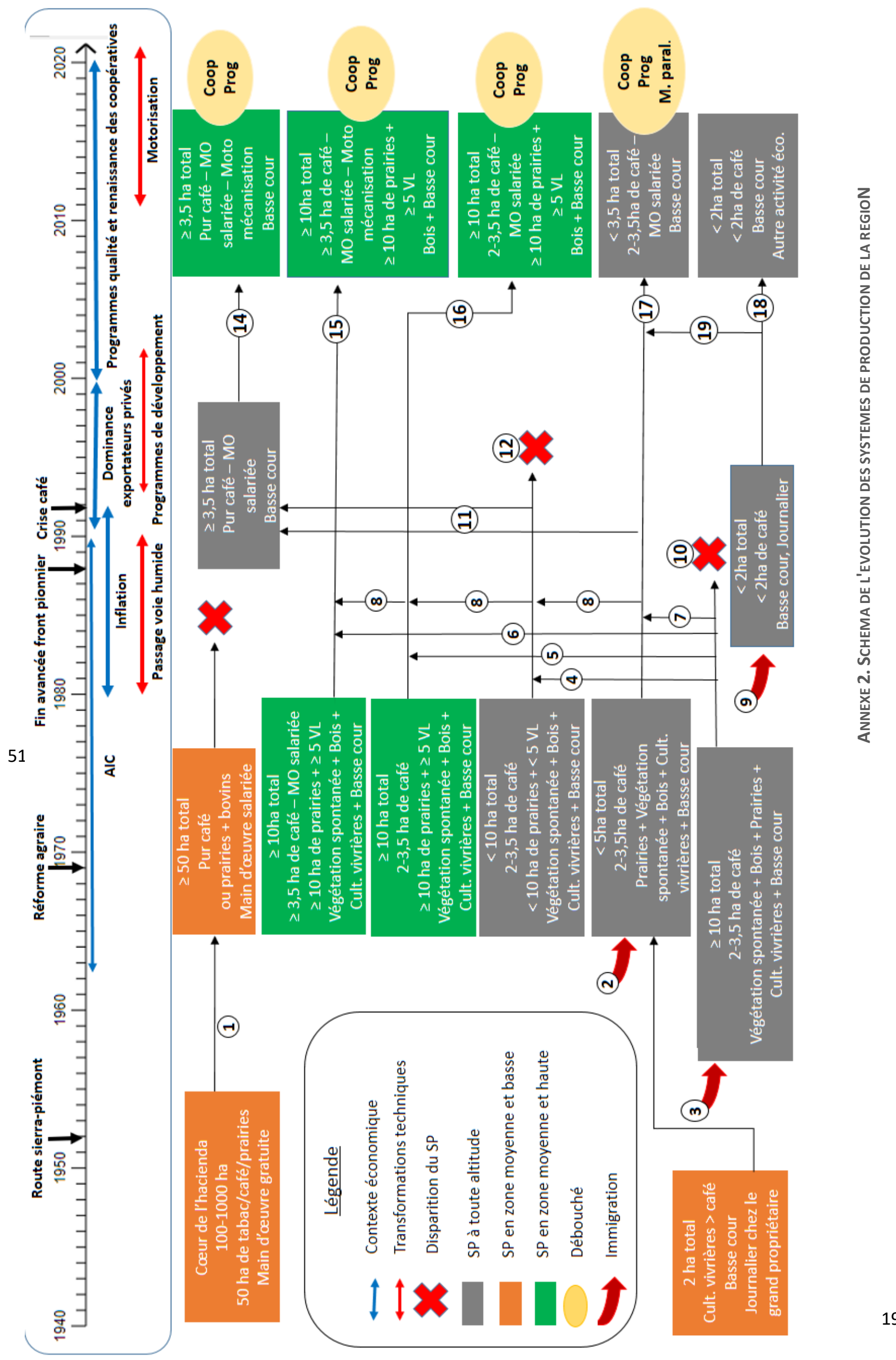

